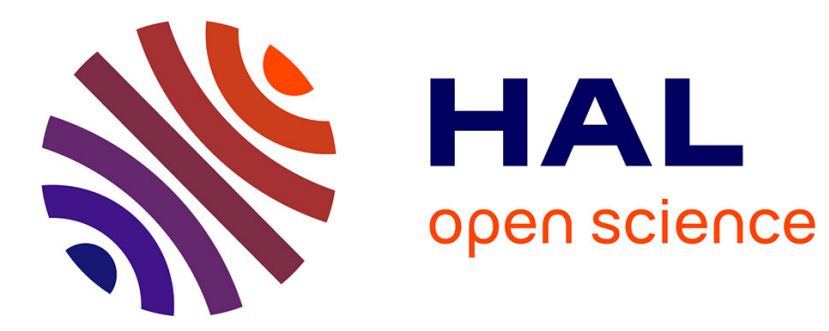

\title{
Two-by-two static, evolutionary and dynamic games
}

Pierre Bernhard, Frédéric Marie Hamelin

\section{To cite this version:}

Pierre Bernhard, Frédéric Marie Hamelin. Two-by-two static, evolutionary and dynamic games. Workshop on Game Theory in Energy, Resources and Environment, Jun 2007, Nice (FR), France. pp.17. hal-00730099

HAL Id: hal-00730099

https://institut-agro-rennes-angers.hal.science/hal-00730099

Submitted on 5 Mar 2013

HAL is a multi-disciplinary open access archive for the deposit and dissemination of scientific research documents, whether they are published or not. The documents may come from teaching and research institutions in France or abroad, or from public or private research centers.
L'archive ouverte pluridisciplinaire HAL, est destinée au dépôt et à la diffusion de documents scientifiques de niveau recherche, publiés ou non, émanant des établissements d'enseignement et de recherche français ou étrangers, des laboratoires publics ou privés. 


\title{
TWO-BY-TWO STATIC, EVOLUTIONARY, AND DYNAMIC GAMES
}

\author{
PIERRE BERNHARD AND FREDÉRIC HAMELIN
}

\begin{abstract}
We discuss related aspects of the simplest possible games, i.e. games where two players have two pure strategies each, and consider static games, population games, a generalization of evolutionary games, and dynamic games.
\end{abstract}

\section{FOREWORD}

Gilles Kahn and I were classmates at École Polytechnique where, in the academic year 1965-1966, he taught me programming (this was in MAGE 2, a translation in French of Fortran 2 I believe, on a punched tape computer SETI PALAS 250), then we met again and became good friends at Stanford University, where he was a computer science student while I was in aeronautics and astronautics. Our paths were to get closer starting in the spring of 1980 when we started planning and, from 1983 on, heading INRIA-Sophia Antipolis together.

Gilles has always believed that game theory was worth pursuing. He was adament that our laboratory should take advantage of my being conversant with that topic. He was instrumental in maintaining it alive in the lab.

He was to be later the president of INRIA who presided over the introduction of "biological systems" as a full-fledged scientific theme of INRIA. Although this was after I had left INRIA, this again met with my personal scientific taste, since I had embraced behavioural ecology as my main domain of interest and of application of dynamic games, much thanks to Eric Wajnberg, from INRA, but also out of an old desire of looking into ecological applications of these techniques.

It is why I think fit to write here a few words about games and behavioural ecology, and also population dynamics and evolution, closely related topics.

Pierre Bernhard

\section{INTRODUCTION}

What follows must be regarded as a distraction with $2 \times 2$ games, the very simple structure of such static games shaping the dynamic aspects of both evolutionary games, - a topic invented by the biologists-, and bilinear differential games, of which we show here an example in behavioural ecology where it arises naturally.

We begin with a short taxonomy of two by two static games, which will be usefull in the sequel. Then we investigate how concepts of evolutionary game theory translate in that simple case. A natural generalization of classical evolutionary

Date: June 29, 2007.

I3S, University of Nice-Sophia Antipolis and CNRS, France. 
games is population games. We develop some very simple, yet probably original, results for such games still in the $2 \times 2$ case, and look at their relationship to evolutionary games.

Then we venture into differential games. The literature on differential games bears a striking difference with that on classical game theory in that, while the latter is mainly concerned with mixed strategies — up to the point that ordinary decisions have to be called pure strategies to recall that they are not mixed-, mixed strategies have had little impact on differential games research. On the one hand, differential games have been mainly concerned with state feedback or non anticipative strategies, and the concept of mixed state feedback, or, for that matter mixed nonanticipative strategy, is surely not simple. On the other hand, most of that literature has considered continous decision sets, as opposed to finite, thus allowing for enough convexity or concavity without relying on mixed strategies.

However, we recently showed ([7]) that in the case of a two-player (non-zerosum) game where each player has only two possible controls - the framework of this article-, not only mixed strategies come up as a natural concept, but moreover they lead to a concept of bi-singular trajectory field which seems to have no counterpart in control theory. Looking into older literature, this concept should have been uncovered in the late 60's or early 70's. We are surprised - and a bit suspicious - that we did not find any mention of it.

We use this theory to investigate a problem of conflict over parental care which has been investigated in the literature on behavioural ecology $([8,11,9,4])$ in various forms (static, discrete dynamic, symmetric...). Here we adopt a continuous time model, asymmetric, that fits with the current paper, and is solved via our theory of bi-singular trajectory field. We find that the solution of the more realistic finite horizon version investigated here, shares, to our surprise, some features of the infinite horizon version investigated in [7], but also displays new features.

\section{STATIC GAMES}

There is a wealth of classical $2 \times 2$ static games, starting with the famous Prisoner Dilemma - a story due to Tucker-, including Stag and Hare, Hawk and Doves, Sex War, - four related games that attempt to capture the benefit of cooperation over agression (See [3]). We propose here a taxonomy of all $2 \times 2$ games adapted to our use in the sequel.

2.1. Notations. Let a two-person game be described by the following $2 \times 2$ bimatrix:

\begin{tabular}{r|rr|rr}
\multicolumn{1}{l}{$u_{1} \backslash u_{2}$} & 1 & & 2 \\
\cline { 3 - 5 } 1 & & $a_{2}$ & & $c_{2}$ \\
1 & $a_{1}$ & & $b_{1}$ & \\
\hline & & $b_{2}$ & & $d_{2}$ \\
2 & $c_{1}$ & & $d_{1}$ & \\
\hline
\end{tabular}

Player 1 chooses the row through his control $u_{1}$, his payments —or rewards- are indexed by the subscript 1 , while player 2 chooses the column through her control $u_{2}$, her payments being indexed by the subscript 2 . 
We shall all along adopt the convention that when a property holds for indices $i=1$ and $i=2$, we shall simply write it with the index $i$ without everytime writing $i \in\{1,2\}$, which shall be implicit. Also, the index $j$ in an expression involving $i$ will mean $j=3-i$.

We let

$$
A_{i}=\left(\begin{array}{cc}
a_{i} & b_{i} \\
c_{i} & d_{i}
\end{array}\right)
$$

Because of the way we have arranged the bi-matrix of payments, each player chooses the line of his or her own matrix, the opponent chooses the column. Let

$$
\delta_{i}=a_{i} d_{i}-b_{i} c_{i}, \quad \sigma_{i}=a_{i}-b_{i}-c_{i}+d_{i},
$$

and when $\sigma_{i} \neq 0$,

$$
p_{j}^{\star}=\frac{d_{i}-b_{i}}{\sigma_{i}}, \quad 1-p_{j}^{\star}=\frac{a_{i}-c_{i}}{\sigma_{i}} .
$$

2.2. Interpretation. We notice that if $\sigma_{i}=0$, player $i$ has a dominating pure strategy since $a_{i}-c_{i}=b_{i}-d_{i}$, so that the first strategy dominates the second one if both are positive, and the second dominates if both are negative. (And his choice is void if both are zero.)

Moreover, we also stress that $\sigma_{i}$ is the second derivative of the restriction of the quadratic form of $\mathbb{R}^{2}$

$$
x \mapsto\left\langle x, \frac{1}{2}\left(A_{i}+A_{i}^{t}\right) x\right\rangle
$$

to the subspace orthogonal to the vector $(11)$. This will have an impact in view of classical theorems on evolutionary stable strategies (ESS).

If we let $p_{i}$ and $1-p_{i}$ be the probabilities that player $i$ chooses his or her first and second pure strategy respectively, $p_{i}^{\star}$ is a candidate strategy of player $i$ equalizing for player $j$, i.e. such that

$$
\left(\begin{array}{ll}
p_{i} & 1-p_{i}
\end{array}\right) A_{j}=\frac{\delta_{j}}{\sigma_{j}}\left(\begin{array}{ll}
1 & 1
\end{array}\right) .
$$

However, $p_{i}^{\star}$ can be a mixed strategy only if it belongs to $[0,1]$.

The property that $p_{i}^{\star} \in(0,1)$ means that player $j$ has a dilemma: the best decision for that player depends on the decision of the other one. Conversely, if either $p_{i}^{\star} \leq 0$ or $p_{i}^{\star} \geq 1$, one line of $A_{j}$ dominates the other one (weakly if $p_{i}^{\star}$ is at one of the bounds), so that player $j$ can play that decision regardless of the opponent's choice. The dominating line is the first one if $\sigma_{j} p_{i}^{\star}<0$, and the second one if $\sigma_{j} p_{i}^{\star}>0$.

Invariance We stress the following invariances and symetries:

- under addition of the same constant to all four entries of $A_{i}, \sigma_{i}$ and $p_{j}$ are invariant.

- interchanging the order of the pure strategies of player $i$, but not $j$, changes both $\sigma_{i}$ and $\sigma_{j}$ to their opposite, leaves $p_{j}^{\star}$ invariant, and, obviously, changes $p_{i}^{\star}$ to $1-p_{i}^{\star}$. 
- In evolutionary games, the sign of $\sigma_{i}$ will have a strong meaning. But this is a symmetric game with $A_{1}=A_{2}$, so that one can only interchange the order of the pure strategies of both players simultaneously, thus preserving this sign.

2.3. Taxonomy. To avoid unnecessary particular cases, we make the following hypothesis:

\section{Hypothesis 1.}

$$
a_{i} \neq c_{i} \quad \text { and } \quad b_{i} \neq d_{i} .
$$

As a consequence both $p_{i}^{\star}$ are different from 0 and 1 .

Let us list the possibilities that arise:

Theorem 1. Under hypothesis 1 , occurences of Nash equilibria in $2 \times 2$ games are as follows:

(1) Any of the $\sigma_{i}=0$. Then, Player $i$ has a dominating pure strategy, the game has a single Nash equilibrium, which is pure.

(2) Both $\sigma_{i} \neq 0$, but one at least of the $p_{i}^{\star} \notin(0,1)$. The corresponding player(s) $j$ has a dominating pure strategy. There is a unique Nash equilibrium, which is in pure strategies.

(3) Both $\sigma_{i} \neq 0$, and both $p_{i}^{\star} \in(0,1)$. Then $\left(p_{1}^{\star}, p_{2}^{\star}\right)$ is a mixed Nash equilibrium. Two subcases arise.

(a) $\sigma_{1} \sigma_{2}<0$. There is no Nash equilibrium in pure strategies. The mixed Nash equilibrium is the only one.

(b) $\sigma_{1} \sigma_{2}>0$. There are two pure Nash equilibria in addition to the mixed one. They are,

(i) if $\sigma_{i}<0,(0,1)$ and $(1,0)$ (in terms of $p_{1}$ and $p_{2}$ ).

(ii) if $\sigma_{i}>0,(1,1)$ and $(0,0)$. (A coordination game, e.g. Sex War.)

\section{Proof}

(1) The case where one $\sigma_{i}=0$ has been covered in the subsection "interpretation" above.

(2) Assume that both $\sigma_{i} \neq 0$, but that $p_{i}^{\star} \notin(0,1)$. (Remember that our hypothesis 1 rules out the case $p_{i}^{\star} \in\{0,1\}$.) Then $p_{i}^{\star}$ and $1-p_{i}^{\star}$ have opposite signs. Therefore, according to (1) $b_{j}-d_{j}$ and $a_{j}-c_{j}$ have the same sign. Thus the first line of $A_{j}$ dominates the second if that common sign is positive, and conversely if it is negative. The domination is strict because of our hypothesis 1 . Therefore, a Nash equilibrium has to be pure for player $j$. But then player $i$ must play his or her best response against that pure strategy, which is pure and unique again due to hypothesis 1 . (If both $p_{i}^{\star}$ are outside $(0,1)$, both players have a dominating strategy. A typical example is Prisoner's Dilemma.)

(3) If both $p_{i}^{\star}$ belong to $(0,1)$, they constitute a pair of mutually equalizing strategies, hence a mixed Nash equilibrium. 
(a) The only two possibilities for there being no pure Nash equilibrium, is that, for a choice of $i$ and $j, a_{i}>c_{i}$ and $d_{i}>b_{i}$ while $a_{j}<c_{j}$ and $d_{j}<b_{j}$. Then, trivially $\sigma_{i}>0$ and $\sigma_{j}<0$.

(b) Otherwise, one of the two pairs of diagonally opposite payments are Nash. The cases $i$. or $i i$. of the theorem can be checked by inspection.

Remark If the context allows one to number the pure strategies of both players independently, then cases $i$ and $i i$ of $3 \mathrm{~b}$ in theorem 1 above are not different, since, according to the facts pointed out in the paragraph "invariance", one is converted into the other one by interchanging the numbering of the pure strategies of any one (but one only) of the players.

\section{EVOLUTIONARY AND POPULATION GAMES}

3.1. Mixed strategies and dynamics. In that section, we investigate the particular case of our simple $2 \times 2$ games, the implications of a simple idea: replace players by populations, and probabilities in mixed strategies by proportions of the population that use a given (pure) strategy. One can still recover a probabilistic interpretation, in that, if an individual is chosen "at random" (with a uniform law among the population), the probabilities that it play one or the other strategy (that it be of one or the other phenotype) agree with population proportions. Yet, each individual uses a unique strategy (is of a given phenotype).

On the one hand, this gives a much more concrete and convincing interpretation of mixed strategies. On the other hand, this allows naturally for an evolution of the mixed strategies as individuals in the population switch from one pure strategy to another one.

In that respect, Sandholm [13] shows that at least two very natural strategy revision schemes lead to the same strategy dynamics, which are those considered in behavioural ecology if the fitness of a phenotype (the payment associated to a pure strategy) is taken to be the growth rate of the sub-population using it.

We use that last explanation to justify the so-called "replicator equation". We imply that a Nash equilibrium is the credible outcome of a game if it is stable under that dynamics. For the sake of completeness, we shall compare it with the Cournot —or pseudo-gradient-dynamics.

\subsection{Evolutionary games.}

3.2.1. Taxonomy. Evolutionary games consider a competition between several (here, two) behaviours within a single population. In our wording, this means that, on the one hand $A_{1}=A_{2}=: A$, and on the other hand, $p_{1}=p_{2}=: p$.

In that context, two remarks are in order concerning the application of theorem 1. On the one hand, the case 3 a cannot appear. On the other hand, interchanging the numbering of the pure strategies of one player alone is not possible, hence the two sub cases of case $3 \mathrm{~b}$ of theorem 1 are indeed different. And the two pure Nash equilibrium of the sub-case $i$ being non symmetric, they are not Nash equilibria in that context. 
The basic concept is that of an Evolutionarily Stable Strategy (ESS) which is a Nash equilibrium in that symmetric context, plus a second order condition, that in the case of a mixed Nash point, strategies as good as $p$ against $p$ be less efficient than $p$ against themselves, which we state here in the simple form it takes for a $2 \times 2$ game

Definition 1. A symmetric strategy $(p, p)$ of a symmetric game is called an ESS if

(1) It is a Nash equilibrium,

(2) If $p \notin\{0,1\}$ (then it has to be $p^{\star}$ of the previous section), for any $q \in[0,1]$ different from $p$,

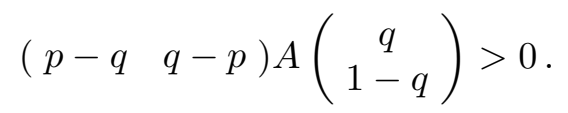

The ESS that may arise are now described by this theorem:

Corollary 1. Occurences of ESS in $2 \times 2$ symmetric games are as follows:

(1) $\sigma=0$. There is a unique ESS, which is pure. (Typically, Prisoner's Dilemma according to [3].)

(2) $\sigma \neq 0$ but $p^{\star} \notin(0,1)$. There is a unique ESS, which is pure. (Typically, Prisoner's Dilemma, with $c<a<d<b$.)

(3) $\sigma \neq 0, p^{\star} \in(0,1)$.

(i) If $\sigma<0$, the only ESS is $p^{\star}$, (typically, Hawk and Dove),

(ii) if $\sigma>0$, there are two pure ESS : $(0,0)$ and $(1,1)$, and no mixed ESS. (Typically, Stag and Hare.)

Proof This is just applying theorem 1 to definition 1 , except for the case $p^{\star} \in(0,1)$ which requires to sort ESS among Nash points. But only symmetric Nash points may be ESS, and a simple calculation shows that

$$
\left(\begin{array}{cc}
p^{\star}-p & p-p^{\star}
\end{array}\right) A\left(\begin{array}{c}
p \\
1-p
\end{array}\right)=-\sigma\left(p-p^{\star}\right)^{2}
$$

this is positive if and only if $\sigma<0$.

Several remarks are in order. The only mixed ESS is therefore obtained when $\sigma<0, p^{\star} \in(0,1)$. Imbedded into a population with that particular mix, individuals using strategy 1 and 2 fare as well. This caracterization of an equilibrium population was first discovered by Wardrop [14].

3.2.2. Dynamics. Assume payments to pure strategies are to be understood as subpopulation growth rates. Let therefore $n_{i}$ be the number of individuals of type $i$ in the population, and $p=n_{1} /\left(n_{1}+n_{2}\right)$. Assume furthermore that the growth rate of each sub-population is

$$
\begin{aligned}
& \frac{\dot{n}_{1}}{n_{1}}=a p+b(1-p) \\
& \frac{\dot{n}_{2}}{n_{2}}=c p+d(1-p) .
\end{aligned}
$$


A straightforward calculation yields the replicator dynamics:

$$
\begin{array}{ll}
\dot{p}=\sigma p(1-p)\left(p-p^{\star}\right) & \text { if } \sigma \neq 0, \\
\dot{p}=(b-d) p(1-p) & \text { if } \sigma=0 .
\end{array}
$$

It is straightforward to check, concerning (3), that if $\sigma<0$, its only stable equilibrium is $p^{\star}$, while both 0 and 1 are stable, and not $p^{\star}$, if $\sigma>0$. This is an instance of the general theorem that states that ESS are (at least locally) stable points of the replicator dynamics. The same holds for (4) which converges to 0 or 1 according to whether $b-d$ is negative or positive.

Stag and Hare's paradox Stag and Hare (after J-J. Rousseau [12]) is a symmetric game with

$$
A=\left(\begin{array}{cc}
D & 0 \\
1 & 1
\end{array}\right)
$$

with $D>1$. This an instance of the case $\sigma=D>0, p^{\star}=1 / D \in(0,1)$, the last case $(3 . b, i i)$ of theorem 1 , where there are three symmetric Nash equilibria, but only two pure ESS. Indeed, if the mixed strategy $p^{\star}$ is considered as a possible outcome of the game, we run into a paradox: the probability of choosing strategy 1 would decrease with the payment of coordinated choices in favor of 1 . The solution is in the replicator dynamics : the interval $\left(p^{\star}, 1\right)$ is the attraction basin of the ESS $(1,1)$. And it is increasing with $D$.

\subsection{Population games.}

3.3.1. Equilibria and stability. We turn now to games between two different populations, each composed of individuals of two different types. This is exactly the framework of (non symmetric) games, but with players interpreted as populations. The status of Nash equilibria are therefore described by theorem 1. We do not attempt to define the equivalent of an ESS, but rely on the stability of the replicator dynamics to select realistic outcomes of a game. As mentioned earlier, several natural considerations lead to this same dynamics, either in a learning paradigm (see [13]) or, as we assume here, in an evolutionary context.

We now have two sets $\left(n_{i 1}, n_{i 2}\right)$ of subpopulations numbers, and we extend equations (2) to both populations, as well as the definition $p_{i}=n_{i 1} /\left(n_{i 1}+n_{i 2}\right)$. Differentiating that last expression, we get, in case both $\sigma_{i} \neq 0$, we find

$$
\dot{p}_{i}=\sigma_{i} p_{i}\left(1-p_{i}\right)\left(p_{j}-p_{j}^{\star}\right) \text {. }
$$

These equations have $(1,1),(1,0),(0,1)$, and $(0,0)$ as equilibria, and if both $p_{i}^{\star} \in$ $(0,1),\left(p_{1}^{\star}, p_{2}^{\star}\right)$. The stability of these equilibria are readily seen from the jacobian

$$
J\left(p_{1}, p_{2}\right)=\left(\begin{array}{cc}
-\sigma_{1}\left(1-2 p_{1}\right) & \sigma_{1} p_{1}\left(1-p_{1}\right) \\
\sigma_{2} p_{2}\left(1-p_{2}\right) & -\sigma_{2}\left(1-2 p_{2}\right)
\end{array}\right) .
$$

We skip the discussion of the four "pure" cases. The conclusion is that the pure Nash equilibria are stable. Let us concentrate on the phase portrait in case 3 of the theorem. The jacobian at $\left(p_{1}^{\star}, p_{2}^{\star}\right)$ is

$$
J\left(p_{1}^{\star}, p_{2}^{\star}\right)=\left(\begin{array}{cc}
0 & \sigma_{1} p_{1}^{\star}\left(1-p_{1}^{\star}\right) \\
\sigma_{2} p_{2}^{\star}\left(1-p_{2}^{\star}\right) & 0
\end{array}\right) .
$$


Therefore two cases arise

(a) $\sigma_{1} \sigma_{2}<0$. The equilibrium $\left(p_{1}^{\star}, p_{2}^{\star}\right)$ is a center,

(b) $\sigma_{1} \sigma_{2}>0$. The equilibrium $\left(p_{1}^{\star}, p_{2}^{\star}\right)$ is a saddle.

Let us furthermore emphasize the following fact. Consider the functions

and

$$
U_{i}\left(p_{i}\right)=p_{i}^{\star} \ln \frac{p_{i}^{\star}}{p_{i}}+\left(1-p_{i}^{\star}\right) \ln \frac{1-p_{i}^{\star}}{1-p_{i}}
$$

$$
V\left(p_{1}, p_{2}\right)=\sigma_{2} U_{1}\left(p_{1}\right)-\sigma_{1} U_{2}\left(p_{2}\right) .
$$

Lemma 1. The function $V\left(p_{1}, p_{2}\right)$ is a first integral of the replicator dynamics (5).

Proof The lemma is proved by direct differentiation, checking that the lagrangian derivatives of the $U_{i}$ 's are $\dot{U}_{i}\left(p_{i}\right)=\sigma_{i}\left(p_{1}-p_{1}^{\star}\right)\left(p_{2}-p_{2}^{\star}\right)$.

3.3.2. Phase portraits. We can now give a fairly complete description of case 3 .

Theorem 2. In case both $\sigma_{i} \neq 0$ and both $p_{i}^{\star} \in(0,1)$,

(a) If $\sigma_{1} \sigma_{2}<0$, the trajectories of the replicator dynamics are all periodic, the center being $\left(p_{1}^{\star}, p_{2}^{\star}\right)$,

(b) If $\sigma_{1} \sigma_{2}>0,\left(p_{1}^{\star}, p_{2}^{\star}\right)$ is a saddle. The two pure Nash equilibria are the stable points of the dynamics. Their attraction basins are separated by the curve $V\left(p_{1}, p_{2}\right)=0$.

Proof It is a classical fact that, as long as the $p_{i}^{\star} \in(0,1)$, the $U_{i}$ are positive, null in $p_{i}^{\star}$ and strictly convex. Hence, if $\sigma_{1}$ and $\sigma_{2}$ are of opposite signs, $V$ is strictly convex or concave, with its extremum 0 in $\left(p_{1}^{\star}, p_{2}^{\star}\right)$, and the trajectories, lying on level curves of $V$, are periodic. Otherwise, the curve $V\left(p_{1}, p_{2}\right)=0$ has to be a trajectory, and as it passes through $\left(p_{1}^{\star}, p_{2}^{\star}\right)$, the result follows.

Take $A_{1}=A_{2}=A$, with $\sigma<0$. Remark that the stable mixed ESS of the previous section has now turned into a saddle. The ESS dynamics was the diagonal dynamics of the current 2D game. And indeed, in the saddle, the diagonal is the trajectory heading towards the saddle point. But in this 2D game, it is highly unstable. Whether the stable case can be taken as such depends on the context. Two identical populations are not the same as a single population.

3.3.3. Wolves and Lynxes. We give here an example of population dynamics taken from a model of intraguild predation. (This is a somewhat formal model. See [1] for a recent review.) In the classical Hawk and Doves game, one investigates the equilibrium between two behaviours in a population of predators competing for preys. Here, we have two differet species of predators, say wolves and lynxes hunting deers. "Dog does not eat dog". In our model, the competition is extraspecific. But we still have two possible behaviours, agressive or pacific, in each population.

In that model, Lynxes are at a trophic level above that of wolves. In particular, if two "pacific" individuals meet, the wolf has a smaller reward, while if two agressive meet, the lynx eats the wolf. Also, we assume that against a pacific (coward) wolf, a pacific lynx may be better off than an agressive one, because the agressive 
individual has spent un necessary time and effort chasing a competitor who would have left anyhow.

The result is the following bi-matrix of rewards:

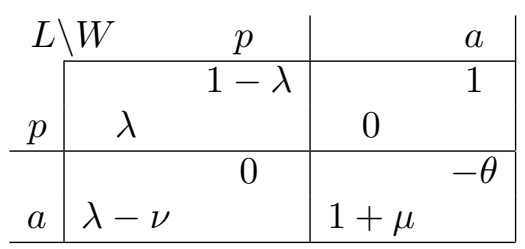

(All greek letters are positive coefficients less than 1.) In that game, we have $\sigma_{1}=1+\mu+\nu$ and $\sigma_{2}=-\lambda-\theta, p_{1}^{\star}=\theta /(\lambda+\theta), p_{2}^{\star}=(1+\mu) /(1+\mu+\nu)$.

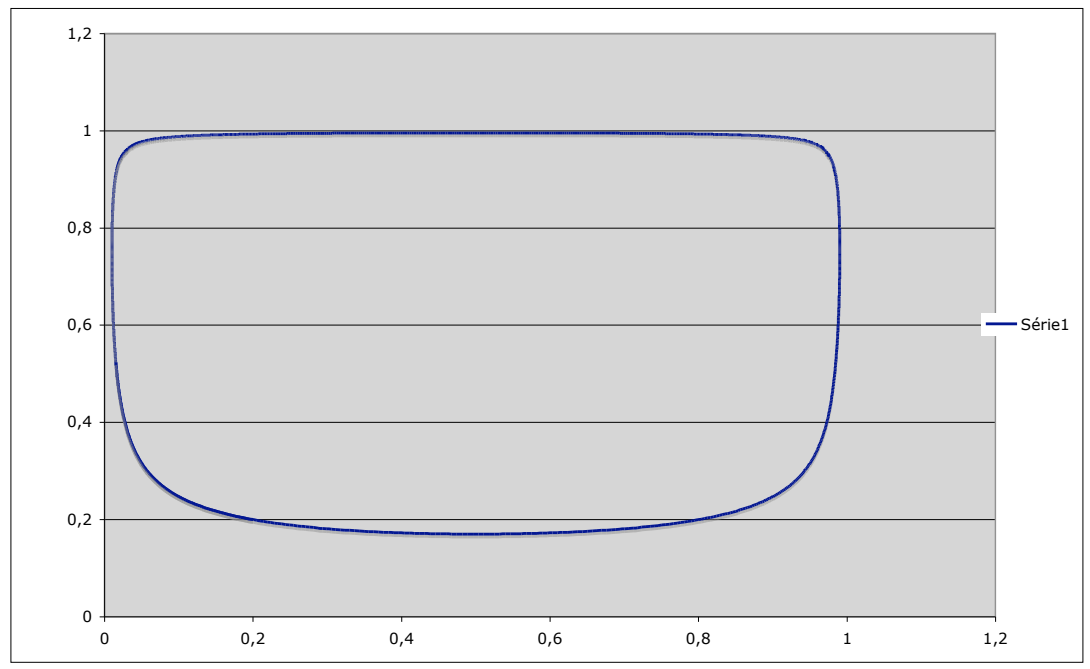

Figure 1. Population dynamics for Wolves and Lynxes, all greek parameters set to $1 / 2$, time span : 40 units.

A typical example of the resulting phase portrait is depicted in Figure 1, where we have taken all "greek" parameters equal to $1 / 2$, initial state at $(0.2,0.2)$. We have integrated with an order 4 Runge Kutta scheme, with a step of $2.5 \times 10^{-2}$ from $t=0$ up to $t=40$.

3.3.4. Cournot Dynamics. It may be worthwhile to compare the replicator dynamics to the "natural" dynamics whereby the $p_{i}$ 's would evolve according to the gradient of the rewards. (This could be considered the natural extension of Cournot iterations, and is Rosen's pseudo-gradient algorithm.) This leads to

$$
\dot{p}_{i}=\sigma_{i}\left(p_{j}-p_{j}^{\star}\right) \text {. }
$$


This is either an harmonic oscillator or a diverging exponential according to the sign of $\sigma_{1} \sigma_{2}$. It does not take into consideration the restriction $p \in[0,1]$ and does not seem to be a good basis for Nash selection.

\section{Conflict over parental care, a differential game}

We turn now to an application of the theory described in $[6,7]$ where we show how a mixed Nash equilibrium of a two-player non-zero-sum game where each player has, as here, two pure strategies (two possible phenotypes), can be found via the solution of a pair of uncoupled PDE's, derived from Isaacs'PDE.

4.1. The parental care game. Rather than an exhaustive taxonomy, which does not seem feasible, we investigate here a variation of a famous problem in behavioural ecology, the conflict over parental care. [2,9]. We significanty improve, we believe, our own treatment of that question in $[6,7]$.

Two animals, 1 and 2, have jointly given birth to an offspring. Each of the two parents may take care of the young, but this time is taken from the time it could spend caring for itself and thus increasing the likelihood of disseminating its genes by other means. Or it may defect. But then the effort put into nestling and gaving birth to he young is wasted. We allow each parent a mixed strategy, in the form of a partial rather than full effort.

Let therefore $x \in \mathbb{R}$ be the weight increase of the young. At initial time, $x=0$. The offspring is adult and viable when $x=1$. But this must happen during the year it was born, say at or before time $T$. Let $u_{i}=1$ if parent $i$ takes care full time of the young, $u_{i}=0$ if it defects. In the "pure" dynamics $\dot{x}$ is given as follows:

\begin{tabular}{c|c|c|}
$u_{1} \backslash u_{2}$ & 0 & 1 \\
\hline 0 & $-\delta$ & $\alpha_{2}$ \\
\hline 1 & $\alpha_{1}$ & $\gamma$ \\
\hline
\end{tabular}

The coefficients $\alpha_{i}, \gamma$ and $\delta$ are all assumed positive, with $\gamma>\alpha_{1}>\alpha_{2}$. We let $\beta=\gamma-\alpha_{1}-\alpha_{2}$ be the synergy coefficient.

Allowing for "mixed strategies" or partial efforts $u_{i} \in[0,1]$ leads to

$$
\begin{gathered}
\dot{x}=a_{1} u_{1}+a_{2} u_{2}+c u_{1} u_{2}-\delta \\
a_{i}=\alpha_{i}+\delta, \quad c=\gamma-\alpha_{1}-\alpha_{2}-\delta .
\end{gathered}
$$

We allow both parents to behave in closed loop, i.e. use controls of the form $u_{i}=\phi_{i}(t, x)$. We shall encounter only constant controls, so that existence of solutions to our dynamic equations is not an issue.

The game ends at $\tau=\min \{t \mid x(t)=1, T\}$ The reward of the parents are $M(x(\tau))=1$ or 0 according to whether the young has achieved viability or not, -i.e. $M(1)=1, M(x)=0 \forall x<1$-, decreased by the cost of caring, say

$$
J_{i}\left(u_{1}(\cdot), u_{2}(\cdot)\right)=M(x(\tau))-\varepsilon_{i} \int_{0}^{\tau} u_{i}(t) \mathrm{d} t .
$$

\subsection{Pure equilibria.}


4.2.1. Constant controls. We notice the following simple facts:

\section{Lemma 2.}

(1) Any effort that does not lead to $x(\tau)=1$ is dominated by 0 .

(2) A parent who cares alone should use the pure strategy $u_{i}=1$.

(3) The best response to $u_{i}=1$ is never $u_{j}=1$ unless $\gamma T=1$.

\section{Proof}

(1) If $M(x(\tau))=0$, the payoff to each parent is negative, or 0 for whichever has used $u_{i}=0$.

(2) If a parent cares alone, to reach $x(\tau)=1$, it needs to achieve

$$
\int_{0}^{\tau}\left(a_{i} u(t)-\delta\right) \mathrm{d} t=1, \quad \Longrightarrow \quad a_{i} \int_{0}^{\tau} u_{i}(t) \mathrm{d} t=1+\delta \tau .
$$

Hence its reward is $J_{i}=1-\left(\varepsilon_{i} / a_{i}\right)(1+\delta \tau)$ which is decreasing with $\tau$. Hence it should strive to minimize $\tau$.

(3) Against $u_{j}=1$, a constant response $u_{i}$ yields $\tau=1 /\left[\left(\gamma-\alpha_{j}\right) u_{i}+\alpha_{j}\right]$ which is decreasing with $u_{i}$, as is $J_{i}=1-\varepsilon_{i} \tau u_{i}$. Hence if $\tau<T$, a $u_{i}<1$ still leads to termination before $T$ and a higher reward.

This simple fact suffices to allow us to investigate pure Nash equilibria. Consider the game space in the $(t, x)$ plane. Draw the lines $x=1-\alpha_{i}(T-t)$, called $\mathcal{L}_{i}$, and $x=1-\gamma(T-t)$ called $\mathcal{L}_{\gamma}$, as in figure 1 . (We carry the discussion below for $x(0)=0$, and with respect to the position of 0 on the time axis. This could easily be extended to an arbitary initial pair $\left(t_{0}, x_{0}\right)$.)

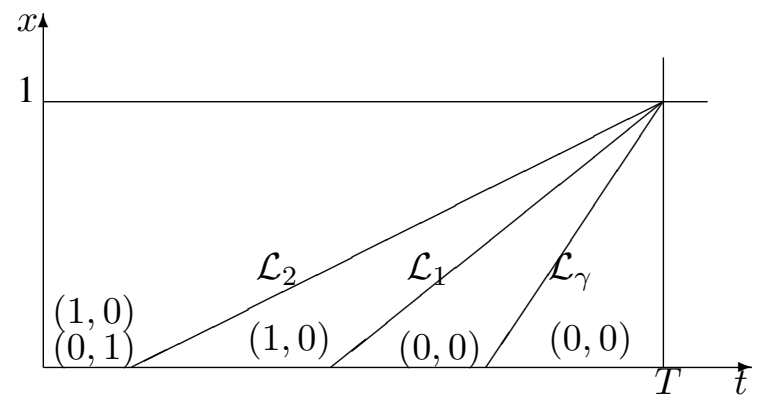

FIGURE 2. The pure Nash equilibria if the $\varepsilon_{i}$ are small.

We claim the following

Theorem 3. The following discussion provides all pure Nash equilibria with constant controls

Discussion To the right of line $\mathcal{L}_{\gamma}$, the child cannot be brought to adulthood within the remaining time. Therefore, the only Nash equilibrium is $(0,0)$.

Assume $\alpha_{1}>\alpha_{2}$. To the right of line $\mathcal{L}_{1}$, no parent can bring the child to adulthood alone. Therefore, if the other parent plays $u_{j}=0$, the optimum is 
$u_{i}=0$, and $(0,0)$ is Nash. A joint effort may drive $x$ to 1 before time $T$, but, according to the lemma, except on the line $\mathcal{L}_{\gamma},(1,1)$ cannot be a Nash equilibrium. We shall see mixed equilibria in that region.

Between lines $\mathcal{L}_{1}$ and $\mathcal{L}_{2}$, the parent 1 can succeed alone. If its reward in so doing is positive, it is its best response against $u_{2}=0$. And of course $u_{2}=0$ is the best response to $u_{1}=1$ since it yields a reward of 1 to parent 2 . Therefore, $(1,0)$ is the only Nash equilibrium if $\varepsilon_{1}<\alpha_{1}$. Otherwise, the same situation as to the right of $\mathcal{L}_{1}$ prevails.

To the left of line $\mathcal{L}_{2}$, both parents are able to succeed alone. Therefore, if both $\varepsilon_{i}<\alpha_{i}$, there are two asymmetric Nash equilibria, $(1,0)$ and $(0,1)$. If any of the $\varepsilon_{i}>\alpha_{i}$, that parent has no incentive to breed the child alone. Therefore, its best response to 0 is 0 . Therefore if one only, say 1 , is in that situation, the only Nash equilibrium is $(0,1)$. If both are, again $(0,0)$ is a Nash equilibrium, and also $(1,1)$ provided that $\varepsilon_{i}<\gamma$.

4.2.2. Synchronous on-off equilibria. If $c>0$, Nash equilibria appear, where both parents care or rest simultanesouly. The following sufficient condition has no claim of optimality. Note that we have used $\alpha_{1} \geq \alpha_{2}$ to keep the most stringent of symmetric conditions.

Theorem 4. Assume $c>0$. Let $\mathcal{T}_{0}$ be a subset of $[0, T]$ with measure $\tau_{0} \leq 1 / \varepsilon_{i}$, $i=1,2$. Assume that the controls $\bar{u}_{1}(t)=\bar{u}_{2}(t)=\mathbb{1}_{\mathcal{T}_{0}}(t)$ generate a trajectory $\bar{x}(t)$ ending at $\bar{x}(\tau)=1$ before time $T$, and that $\left(1, \bar{u}_{2}\right)$ generate a trajectory ending at $\tau_{1}$. Assume further that over $\left[\tau_{1}, \tau\right]$, the trajectory $\bar{x}(t)$ lies below the line of slope $\gamma-\alpha_{2}$ passing through its end-point. Then the pair $\left(\bar{u}_{1}, \bar{u}_{2}\right)$ is a Nash equilibrum.

Proof Fix $u_{2}=\bar{u}_{2}$, and pick an arbitrary $u_{1}(\cdot)$. If the pair $\left(u_{1}(\cdot), \bar{u}_{2}\right)$ does not lead to termination before time $T$, parent 1 incurs a negative reward, while the condition $\tau_{0} \leq 1 / \varepsilon_{1}$ insures a positive reward for the pair $\left(\bar{u}_{1}, \bar{u}_{2}\right)$. Let therefore $\tau^{\prime}$ be the termination time on this new trajectory. Note that, necessarily, $\tau^{\prime} \geq \tau_{1}$. Two cases arise depending on whether $\tau^{\prime}$ is less or more than $\tau$.

If $\tau^{\prime}<\tau$, the support of $\bar{u}_{2}$ may have been curtailed by the early termination. Let $\mathcal{T}_{2}$ be that curtailed support, and $\tau_{2}$ its measure. Let $\mathcal{T}_{1}=\left[0, \tau^{\prime}\right]-\mathcal{T}_{2}$, and let $v_{1}$ and $w_{1}$ be the integrals of $u_{1}(\cdot)$ respectively over $\mathcal{T}_{1}$ and $\mathcal{T}_{2}$. We have

$$
x\left(\tau^{\prime}\right)=1=a_{1}\left(v_{1}+w_{1}\right)+a_{2} \tau_{2}+c w_{1}-\delta \tau^{\prime}=\bar{x}(\tau)=\left(a_{1}+a_{2}+c\right) \tau_{0}-\delta \tau .
$$

This can be rearranged in

$$
\left(a_{1}+c\right)\left(v_{1}+w_{1}-\tau_{0}\right)=c v_{1}+a_{2}\left(\tau_{0}-\tau_{2}\right)-\delta\left(\tau-\tau^{\prime}\right) .
$$

The hypothesis in the theorem can be written, using $\gamma-\alpha_{2}=a_{1}+c$,

$$
\bar{x}\left(\tau^{\prime}\right)=\left(a_{1}+a_{2}+c\right) \tau_{2}-\delta \tau^{\prime} \leq\left(a_{1}+a_{2}+c\right) \tau_{0}-\delta \tau-\left(a_{1}+c\right)\left(\tau-\tau^{\prime}\right),
$$

which can be rearranged into

$$
a_{2}\left(\tau_{0}-\tau_{2}\right)-\delta\left(\tau-\tau^{\prime}\right) \geq\left(a_{1}+c\right)\left[\tau-\tau^{\prime}-\left(\tau_{0}-\tau_{2}\right)\right] .
$$


Combining this with (7), and noting that necessarily, $\tau_{0}-\tau_{2} \leq \tau-\tau^{\prime}$, we get

$$
\left(a_{1}+c\right)\left(v_{1}+w_{1}-\tau_{0}\right) \geq c v_{1} \geq 0 .
$$

Since $J_{1}\left(u_{1}(\cdot), \bar{u}_{2}\right)-J_{1}\left(\bar{u}_{1}, \bar{u}_{2}\right)=-\varepsilon_{1}\left(v_{1}+w_{1}-\tau_{0}\right)$, we conclude that $J_{1}$ has increased in the change.

Otherwise, if $\tau^{\prime} \geq \tau$, then $\tau_{2}=\tau_{0}$, and (7) directly yields he desired result.

4.3. Time sharing equilibria. If $\beta<0$, that is $\gamma<\alpha_{1}+\alpha_{2}$, i.e. if no synergy exists between the parents, but to the contrary a law of diminishing return prevails, another family of Nash equilibria shows up, where the parents agree to take their turn in caring for the child. Assume that $\alpha_{1} T>1$. Pick a time $\tau<T$ such that $\alpha_{2} \tau<1<\alpha_{1} \tau$. Let

$$
\tau_{1}=\frac{1-\alpha_{2} \tau}{\alpha 1-\alpha_{2}} \quad \text { and } \quad \tau_{2}=\frac{\alpha_{1} \tau-1}{\alpha 1-\alpha_{2}} .
$$

This way, $\tau_{1}+\tau_{2}=\tau<T$ and $\alpha_{1} \tau_{1}+\alpha_{2} \tau_{2}=1$. Choose a partition of $[0, \tau]$ into two (measurable) sets $\mathcal{T}_{1}$ and $\mathcal{T}_{2}$ of respective Lebesgue measures $\tau_{1}$ and $\tau_{2}$. Choose $\bar{u}_{i}(t)=\mathbb{1}_{\mathcal{T}_{i}}(t)$, i.e. 1 if $t \in \mathcal{T}_{i}, 0$ elsewhere.

We claim

Theorem 5. If $\beta<0$, and if both $\varepsilon_{i} \tau_{i}<1$, the pair $\left(\bar{u}_{1}, \bar{u}_{2}\right)$ is a Nash equilibrium.

Proof Fix $\bar{u}_{2}$, and choose an arbitrary $u_{1}(\cdot)$. Let $\tau^{\prime}$ be the time when the game ends, $\mathcal{T}_{2}^{\prime}$ of measure $\tau_{2}^{\prime} \leq \tau_{2}$ the support of $\bar{u}_{2}$ in $\left[0, \tau^{\prime}\right]$-it might be less than $\tau_{2}$ if the game ends earlier - and $\mathcal{T}_{1}^{\prime}$ of measure $\tau_{1}^{\prime}$ its complement. Let also $v_{1}$ and $w_{1}$ be the integrals of $u_{1}(\cdot)$ over $c T_{1}^{\prime}$ and $\mathcal{T}_{2}^{\prime}$ respectively. Notice that $v_{1} \leq \tau_{1}^{\prime}$.

If $\left(u_{1}(\cdot), \bar{u}_{2}\right)$ do not bring the state to 1 before time $T, J_{1}$ is negative. Otherwise, using $v_{1}+w_{1}=\int u_{1} \mathrm{~d} t$,

$$
J_{1}\left(u_{1}(\cdot), \bar{u}_{2}\right)-J_{1}\left(\bar{u}_{1}, \bar{u}_{2}\right)=-\varepsilon_{1}\left(v_{1}+w_{1}-\tau_{1}\right) .
$$

Also, writing the dynamics in terms of the greek parameters, we have that

$$
x\left(\tau^{\prime}\right)=\left(\alpha_{1}+\delta\right) v_{1}+\alpha_{2} \tau_{2}^{\prime}+\left(\gamma-\alpha_{2}\right) w_{1}-\delta \tau_{1}^{\prime}=1=\alpha_{1} \tau_{1}+\alpha_{2} \tau_{2} .
$$

Using the second and the fourth terms of this equality, we easily get that

$$
\alpha_{1}\left(v_{1}+w_{1}-\tau_{1}\right)=\delta\left(\tau_{1}^{\prime}-v_{1}\right)-\beta w_{1}+\alpha_{2}\left(\tau_{2}-\tau_{2}^{\prime}\right) .
$$

If $\beta<0$, the right hand side is positive, hence the variation in $J_{1}$ is negative.

Notice that, contrary to the mixed equilibrium of the next paragraph, this is a strict Nash equilibrium, as the right hand side above can be zero only if $u_{1}=\bar{u}_{1}$.

\subsection{Mixed equilibria.}

4.4.1. Time unconstrained trajectories. We now turn to mixed equilibria, using the theory of [7]. The Isaacs equation is as follows. We let $V_{i}(t, x)$ be the two Value functions of the players. We write $\lambda_{i}(t, x)$ for their derivative in $x$. If they are of class $C^{1}$, they satisfy

$$
\frac{\partial V_{i}(t, x)}{\partial t}+\mathcal{H}_{i}\left(\phi_{1}^{\star}, \phi_{2}^{\star}\right)=0, \quad V_{i}(\tau, x)=M(x),
$$


with

$$
\mathcal{H}_{i}\left(u_{1}, u_{2}\right)=\lambda_{i}\left(a_{1} u_{1}+a_{2} u_{2}+c u_{1} u_{2}-\delta\right)-\varepsilon_{i} u_{i}
$$

In these equations, $\left(\phi_{1}^{\star}, \phi_{2}^{\star}\right)$ stands for a Nash equilibrium of the $2 \times 2$ game whose payoffs are the $\mathcal{H}_{i}$.

It is useful to rewrite this as

$\mathcal{H}_{i}\left(u_{1}, u_{2}\right)=\left(\begin{array}{ll}u_{i} & 1-u_{i}\end{array}\right) \lambda_{i} A\left(\begin{array}{c}u_{j} \\ 1-u_{j}\end{array}\right)-\varepsilon_{i} u_{i}=\left(\begin{array}{cc}u_{i} & 1-u_{i}\end{array}\right) H_{i}\left(\begin{array}{c}u_{j} \\ 1-u_{j}\end{array}\right)$

with

$$
A=\left(\begin{array}{cc}
\gamma & \alpha_{1} \\
\alpha_{2} & -\delta
\end{array}\right), \quad H_{i}=\lambda_{i} A-\varepsilon_{i}\left(\begin{array}{cc}
1 & 1 \\
0 & 0
\end{array}\right) .
$$

As a result, the Nash point sought is that of the bi-matrix game

\begin{tabular}{ccc|cc|}
\multicolumn{1}{c}{$u_{1} \backslash u_{2}$} & 1 & \multicolumn{1}{c}{0} \\
\cline { 2 - 5 } 1 & $\lambda_{1} \gamma-\varepsilon_{1}$ & $\lambda_{2} \gamma-\varepsilon_{2}$ & $\lambda_{2} \alpha_{1}$ \\
\hline & & $\lambda_{1} \alpha_{1}-\varepsilon_{1}$ & \\
\hline 0 & $\lambda_{1} \alpha_{2}$ & $\lambda_{2} \alpha_{2}-\varepsilon_{2}$ & $-\lambda_{2} \delta$ & $-\lambda_{1} \delta$ \\
\hline
\end{tabular}

Notice that in this game, with reference to the notations of the previous sections,

$$
\sigma_{i}=\lambda_{i} c, \quad \Delta_{i}:=\operatorname{det} H_{i}=-\lambda_{i}^{2}\left(a_{1} a_{2}+c \delta\right)+\lambda_{i} a_{j} \varepsilon_{i} .
$$

The Nash equilibria of the above bi-matrix game are singuar controls in the sense of control theory. They are

$$
\phi_{i}^{\star}=\frac{\varepsilon_{j}-\lambda_{j} a_{j}}{\lambda_{j} c}
$$

We investigate a field of trajectories reaching the boundary $x=1$. On such trajectories, locally, the final time is unconstrained. As the rest of the formulation is time invariant, the associated Value is stationary, and $\partial V_{i} / \partial t=0$. Placing this and (10) in (8) yields

$$
\phi_{i}^{\star}=\frac{\delta}{a_{i}}
$$

and therefore

$$
\dot{x}=\delta \frac{a_{1} a_{2}+c \delta}{a_{1} a_{2}}=\delta \frac{\alpha_{1} \alpha_{2}+\gamma \delta}{\left(\alpha_{1}+\delta\right)\left(\alpha_{2}+\delta\right)} .
$$

This slope is necessarily positive and less than $\gamma$. However, depending on $c$, it may be more or less than $\alpha_{i}$.

Theorem 6. If

$$
T>\frac{a_{1} a_{2}}{\delta\left(a_{1} a_{2}+c \delta\right)}, \quad \text { and } \quad \varepsilon_{i}<\frac{a_{1} a_{2}+c \delta}{a_{j}},
$$

the mixed strategies (11) are a Nash equilibrium over feedback strategies. 
Proof Using (12), the first condition in the theorem insures that $\tau<T$, hence $M(x(\tau))=1$, and using this, the second one insures that both parents get a positive reward. (Otherwise, $u_{i}=0$ is better.) If so, the functions

$$
V_{i}(x)=1-\frac{\varepsilon_{i} a_{j}}{a_{1} a_{2}+c \delta}(1-x)
$$

satisfy equations (8) in the region of the game space covered by the trajectories (12), which includes the initial sate of interest, $x(0)=0$.

4.4.2. Time constrained trajectories. We investigate now trajectories that end up exactly at time $T$ with $x(T)=1$, such that both parents get a positive reward. Let $u_{i} \in[0,1]$ be such that

$$
T\left[a_{1} u_{1}+a_{2} u_{2}+c u_{1} u_{2}-\delta\right]=1, \quad T \varepsilon_{i} u_{i}<1 .
$$

Theorem 7. Under conditions (13) the pair of constant controls $\left(u_{1}, u_{2}\right)$ is a Nash equilibrium over feedback strategies if and only if both $u_{i} \geq \phi_{i}^{\star}$ as given by (11).

Proof We compare the constant control $u_{i}$ to any $u_{i}+v_{i}(t)$, assuming that the other parent keeps its control $u_{j}$ constant. Let $\tau$ be the final time on the trajectory generated by these new controls. If $\tau=T$ and $x(T)<1$, both parents have a negative payoff. Parent $i$ looses in so doing. Therefore, the new control can be better only if $\tau \leq T$. Let $w_{i}=\int_{0}^{\tau} v_{i}(t) \mathrm{d} t$.

We have

$$
\tau\left[a_{1} u_{1}+a_{2} u_{2}+c u_{1} u_{2}-\delta\right]+\left(a_{i}+c u_{j}\right) w_{i}=1,
$$

We assume that indeed $\tau \leq T$, thus that $w_{i}>0$. (Recall that $a_{i}+c u_{j} \geq 0$, even though $c$ may be negative.) We have also $J_{i}\left(u_{i}+v_{i}, u_{j}\right)=1-\varepsilon_{i} \tau u_{i}-\varepsilon_{i} w_{i}$, Using (13) and (14) we find that

$$
J_{i}\left(u_{i}+v_{i}, u_{j}\right)-J_{i}\left(u_{i}, u_{j}\right)=-\varepsilon_{i} w_{i} T\left(a_{j} u_{j}-\delta\right) .
$$

Therefore, if $a_{j} u_{j}-\delta<0$, the open loop control $u_{i}+v_{i}(\cdot)$ improves the reward of player $i$, and $\left(u_{1}, u_{2}\right)$ was not Nash. Conversely, if $a_{j} u_{j}-\delta \geq 0$, no open loop control can improve $J_{i}$, and then no feedback strategy can either. (Just apply the above calculation with $u_{i}+v_{i}(t)$ equal to the control of player $i$ generated by a test closed loop strategy and $u_{j}$.) Notice also that if $u_{j}=\phi_{j}^{\star}$, the variation in $J_{i}$ is identically 0 . This is the classical equalization property of mixed Nash equilibria.

The trajectories generated by these new Nash strategies are straight lines through the point $t=T, x=1$. They fill the void between the last bi-singular trajectory and the curve $\mathcal{L}_{\gamma}$ of Figure 2.

4.5. Biological implications. There are several biological considerations to be drawn from this analysis. Let us mention just a few ones.

First, let us comment on the parameters of the game. As opposed to previous literature, we have both a fixed level of welfare to reach and a maximum end time. Moreover, we let welfare go down if the child is left careless. Also, we allow male and female to be asymmetric in terms of "cost" of breeding their offspring. One of the two, for instance, might be more prone to predation, either because it is more 
visible, or less apt to defend its life. Also, we let them differ in their efficiency at gathering food or otherwise breeding the child.

In that respect, intuitively, if $\gamma>\alpha_{1}+\alpha_{2}$, we may consider this as a synergetic effect, since both parents acting together do better than the sum of their lone efforts. But if we consider that the efficiency of a parent is in replacing a decrease rate of $\delta$ by an increase of $\alpha_{i}$, i.e. $a_{i}=\alpha_{i}+\delta$, and similarily for the pair $\gamma+\delta$, then the measure of synergy is rather $c$. Both play a role in the above results.

We do not claim to have described all Nash equilibria. But they are clearly highly non unique. More analysis in terms of biological interpretations is needed to sort them out. We give here a few hints.

We notice that some regions of the game space have the mixed stratey as their natural outcome. It is particularily so if $T$ is large and the $\varepsilon_{i}$ small enough, so that the pure Nash are $(1,0)$ an $(0,1)$. Then, the mixed equilibrium appears as the "fair" outcome. The link with an ESS in a population comprising both males and females remains to be investigated further.

The peculiarity of the mixed Nash is that each parent does exactly the effort which, if made alone, keeps $\dot{x}=0$. The interpretation is that this is true on locally time unconstrained trajectories. Therefore the same reasoning as in [7] holds. The fact that the available time be, globally, constrained by $T$ is reflected, on the one hand, through the possible overlap of the bi-singular field of trajectories with the field $(0,0)$, and on the other hand, by the existence of a new field of mixed equilibria trajectories, filling the gap between the bi-singular field and the fastest trajectory to just-in-time completion of the breeding process.

A last point we want to raise is that of the incentive to defect. It follows from the threshold $\varepsilon_{i}<a_{i}+c \delta / a_{j}$ that, if $c>0$, increasing the efficiency of the partner $j$ will eventually lead to a choice for $i$ to desert. An apparent paradox. The explanation we propose is that $c>0$ means a large synergetic effect. In that case, a less efficient mate, having a lower $a_{j}$, has a larger $\phi_{j}^{\star}=\delta / a_{j}$. (The threshold is precisely $a_{i}+c \phi_{j}^{\star}$.) Thus, under the mixed strategy, it will be more often present in the nest, and through the synergetic effect, this will compensate and over for its lower efficiency.

Is this a plausible explanation for the paradox of the handicap $[5,15,10]$ in sexual selection?

\section{REFERENCES}

[1] Bampfylde C.J. and Lewis M.A.: "Biological control through intraguild predation: case studies in pest control, invasive species and range expansion". Bulletin of Mathematical biology 69 , 1031-1066, 2007.

[2] Clutton-Brock, T.H. The Evolution of Parental Care, University Press, Oxford, 1991.

[3] Doebeli M. and Hauert C.: "Models of cooperation based on Prisoner's dilemma and Snowdrift games", Ecology Letters, 8, pp 748-756, 2005.

[4] Ewald C-O., McNamara J.M., and Houston A.: "Parental care as a differential game: a dynamic extension of the Houston-Davis game.",to appear.

[5] Fisher R.A. The Genetical Theory of Natural Selection, University Press, Oxford, 1930.

[6] Hamelin F.: "Jeux dynamiques en écologie du comportement", thèse de doctorat, Université de Nice, soutenue le 4 juillet 2007. 
[7] Hamelin F. and Bernhard P.: "Uncoupling Isaacs'equations in two-player nonzero-sum differential games. Parental conflict over care as an example". Submitted.

[8] Houston A.I. and Davies N.B.: "The evlution of cooperation and life history in the dunnock Prunella modularis". In Behavioural Ecology, R.M Silby and R.H. Smith eds., pp 471-487, Blacwell Scientific Publications, 1985.

[9] Houston A.I., Szekely T., and McNamara J.M.: "Conflict between parents over care", Trends in Ecology and Evolution 20, pp 33-38, 2005.

[10] Kokko, H., Jennions, M. D., and Brooks, R., "Unifying and testing models of sexual selection" Annual Reviews of Ecology, Evolution and Systematics 37, pp 43-66, 2006.

[11] McNamara J.M. et al.: "A dynamic game-theoretic model of parental care". Journal of Theoretical Biology 205, pp 605-623, 2000.

[12] Rousseau J-J.: Discours sur l'origine et les fondemens de l'inégalité parmi les hommes, Dijon, 1755.

[13] Sandholm W.H.: "Population Games and Evolutionary Dynamics". Preprint, http: //www.ssc.edu/ whs/book/index.html, 2007.

[14] Wardrop J.G.: "Some theoretical aspects of road trafic", Proceedings of the Institute of Civil Engineer, Part II 1, pp 325-378, 1952.

[15] Zahavi, A. "Mate selection — a selection for the handicap", Jal of Theoretical Biology 53, pp 205-214, 1975. 